\title{
Conscious Sedation and Awake Fiberoptic Intubation in a Patient with Difficult Mask Ventilation-A Case Report
}

\author{
Bidur Kumar Baral1,2, Susumu Ide ${ }^{\text {* }}$, Masahiro Uzawa1, Kenkichi Kiyosawa1, \\ Hiroyuki Sasaki ${ }^{3}$, Mikito Kawamata ${ }^{1}$ \\ ${ }^{1}$ Department of Anesthesiology and Resuscitology, Shinshu University School of Medicine, Matsumoto, Japan \\ ${ }^{2}$ Department of Anesthesiology and Critical Care, Norvic International Hospital, Kathmandu, Nepal \\ ${ }^{3}$ Department of Radiology, Shinshu University Hospital, Matsumoto, Japan \\ Email: ${ }^{*}$ ide@shinshu-u.ac.jp
}

Received 28 August 2015; accepted 27 September 2015; published 30 September 2015

Copyright (C) 2015 by authors and Scientific Research Publishing Inc.

This work is licensed under the Creative Commons Attribution International License (CC BY). http://creativecommons.org/licenses/by/4.0/

c) (i) Open Access

\begin{abstract}
Since maxillofacial malignancy is a common cause of facial defects and disfigurement of the face that may make fitting of a mask difficult and cause air leakage from the side, thus making mask ventilation difficult. In addition, distorted anatomy of the airway and base of the skull in such patients may cause difficult intubation (DI). We experienced a case with a huge facial defect due to maxillary carcinoma, in which difficult mask ventilation (DMV) and DI were predicted. After evaluation by three-dimensional airway computed tomography, the airway was secured with conscious sedation using dexmedetomidine, and awake fiberoptic intubation was safely performed. Three-dimensional airway computed tomography seems to be a good tool for successful intubation when DMV and DI are predicted.
\end{abstract}

\section{Keywords}

Conscious Sedation, Difficult Mask Ventilation, Awake Fiberoptic Intubation

\section{Introduction}

Difficult airway is still a challenge for anesthesiologists. It includes difficult intubation (DI), difficult mask ventilation (DMV) or both. Since mask ventilation is a basic fundamental skill in airway management, every anesthesiologist should acquire skills for mask ventilation and should be knowledgeable about the causes of DMV

${ }^{*}$ Corresponding author.

How to cite this paper: Baral, B.K., Ide, S., Uzawa, M., Kiyosawa, K., Sasaki, H. and Kawamata, M. (2015) Conscious Sedation and Awake Fiberoptic Intubation in a Patient with Difficult Mask Ventilation-A Case Report. Open Journal of Anesthesiology, 5, 206-210. http://dx.doi.org/10.4236/ojanes.2015.59037 
and consider alternative options including oropharyngeal airway, nasopharyngeal airway, laryngeal mask airway, transtracheal jet ventilation, cricothyrotomy, and awake fiberoptic intubation (AFOI) when the mask ventilation is difficult [1]. Mask ventilation is the first step of airway management before endotracheal intubation or insertion of any airway devices. It is a rescue technique when endotracheal intubation has failed or has become difficult. Therefore mask ventilation became a major step in any difficult airway algorithm. Every Anesthesiologist should also concentrate on prediction of DI. Prediction of DMV and concomitant DI may be the most difficult situation for anesthesiologists. Here we present a case of a huge maxillofacial defect due to squamous cell carcinoma of the maxilla. After evaluation by three-dimensional airway computed tomography, we managed the airway with conscious sedation using dexmedetomidine and AFOI.

\section{Case Summary}

A 58-year-oldmale, height $168 \mathrm{~cm}$, weight $45 \mathrm{~kg}$, and American Society of Anesthesiologists physical status 1 , was presented with a history of a large wound on the left side of his face and swelling. He was diagnosed with squamous cell carcinoma of the maxilla 4 years ago and had been treated with chemotherapy and radiotherapy. He underwent extended total maxillectomy 3 years ago. His wound laterbecame infected, and chronic osteomyelitis of the mandible developed. He was diagnosed as having chronic osteomyelitis of the left mandible with large facial defect on left side of face. He was planned for debridement, sequestrectomy and reconstruction of the defect with anterolateral free flap of the thigh. On examination, his vital parameters were within normal ranges. His general physical examination and systemic examination were unremarkable. Results of laboratory tests were within normal ranges.

Airway assessment showed that mouth opening was restricted (less than 1 finger). He had a large ulcerated wound on the left side of his face that extended from the left supra orbit to lower jaw and involved the eye. Large amounts of tissue and muscle were lost on the left cheek (Figure 1(a)). A mandibular protrusion test could not be performed due to pain. Neck movement was normal and thyromental distance was $6.5 \mathrm{~cm}$.

The surgery was plannedunder general anesthesia. Considering the DMV (Figure 1(b)), we had planned to secure the airway with AFOI under conscious sedation. DI was also predicted because of distorted anatomy of the airway and base of the skull. Three-dimensional computed tomography (3D-CT) of the airway was obtained before surgery, and access to the trachea was pre-evaluated for tracheal intubation (Figure 1(c)). Finally, we planned nasal intubation at the right side using AFOI. The procedure for AFOI was explained in detail to the patient and patient's family and informed consent was obtained. The patient was kept nil per oral for 8 hours prior to surgery.

On the day of surgery, the patient was transferred to the operation theatre, monitors (i.e. electrocardiogram, non-invasive blood pressure, pulse oximeter, temperature probe) were connected. An intravenousline was secured with an $18 \mathrm{G}$ intravenous cannula. Arterial cannulation was done on his left hand for invasive blood pressure monitoring. The patient was catheterized with a Foley catheter. The availability and working condition of all components of the difficult airway trolley were checked. Arescue ventilation device was prepared, and standby arrangement for emergency tracheostomy was also prepared. For nasal decongestion and anesthesia, the right nasal cavity was packed with ribbon gauze soaked in $1 \%$ lidocaine and epinephrine solution $(1: 200,000)$ and was leftin place for 10 minutes. For conscious sedation, injection dexmedetomidine at $6 \mathrm{mcg} / \mathrm{kg} / \mathrm{hr}$ was started and sedation was assessed with the Richmond agitation-sedation scale (RASS) [2]. After 10 minutes of dexmedetomidine infusion, the RASS score was 0 to -1 , and the dose of dexmedetomidine was decreased to 0.4 $\mathrm{mcg} / \mathrm{kg} / \mathrm{hr}$. There was no hypotension or bradycardiaduring the infusion of dexmedetomidine.

After 10 minutes, the gauze pack was removed, and the nasal passage was lubricated with $2 \%$ lidocaine jelly. Serial dilation of the nasal cavity was performed using nasopharyngeal airways with internal diametersof 6, 7 and $8 \mathrm{~mm}$. A Phycon Reinforced Endotracheal tube (ET tube; Fuji Systems Corporation, Tokyo, Japan) with an internal diameter of $7 \mathrm{~mm}$ was gently passed through the right nasal cavity into the hypopharynx. There was no cough orgrimace. Then a fiberoptic bronchoscope (TOKIBO-Ambu a Scope 3, Tokyo, Japan) was inserted through the ET tube. The patient was able to respond and was instructed to take slow and deep breaths. The epiglottis and vocal cords were visualized, and an attempt was made to insert the ET tube. When the ET tube reached the vocal cord, the patient started coughing. After waiting for opening of the vocal cord, $4 \%$ lidocaine was sprayed to the vocal cord through the port of the bronchoscope. When the vocal cord was opened, intubation proceeded without difficulty. The position of the ET tube was further confirmed by checking bilateral equal air 


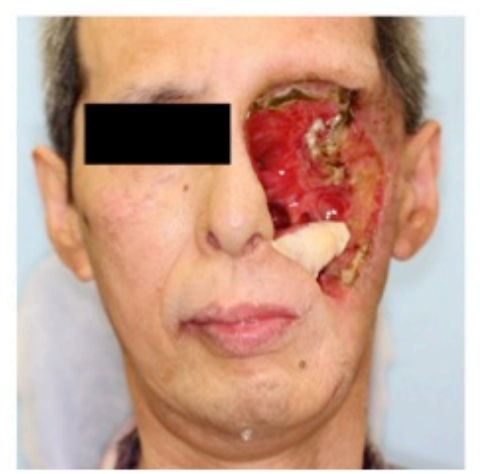

(a)

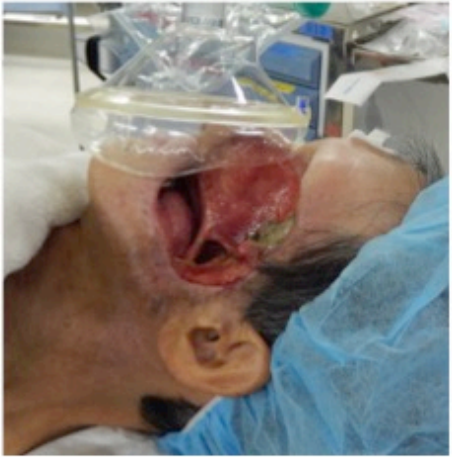

(b)

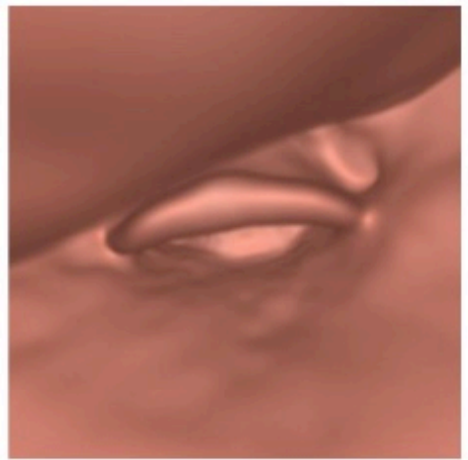

(c)

Figure 1. Large ulcerated wound on the left side of the face (a), difficult mask ventilation (b), and three-dimensional computed tomography (3D-CT) of the airway (c).

entry and end-tidal $\mathrm{CO}_{2}$ tracing. The ET tube was then fixed. Sixty mg of propofol was injected intravenously, and eyelash reflex was noted. Thirty mg of rocuronium was injected intravenously after the disappearance of eyelash reflex. Then dexmedetomidine infusion was stopped and the patient was kept on a mechanical ventilator, allowing the surgeon to start the surgery. The patient was maintained intraoperatively with oxygen, remifentanil, rocuronium and sevoflurane. The patienttolerated the procedure well, and he had no recall of fiberoptic intubation.

Surgery lasted for 13 hours, and the intraoperative period was uneventful. Total blood loss was $300 \mathrm{ml}$. After completion of surgery, the patient was transferred to the intensive care unit and kept on mechanical ventilation with propofol sedation. We did not extubated the patient because of anticipation of glottic edema, anticipated DI and long surgery. The patient was successfully extubated on the next day. The postoperative period was uneventful, and the patient was discharged after two weeks.

\section{Discussion}

Mask ventilation is the initial and mostessential step in airway management. Every anesthesiologist should acquire the skills for mask ventilation [1]. DMV is a situation in which it is not possible for the anesthesiologist to provide adequate face mask ventilation due to an inadequate mask seal, excessive gas leakage or excessive resistance to ingress or egress of gas [3]. There is a wide variation in the incidence of DMV. The incidence of DMV was reported to be $5 \%$ by Langeron et al. [4] and $1.4 \%$ by Asai et al. [5]. However, DMV is underestimated by most anesthesiologists. DMV may occur before intubation or after failure of endotracheal intubation [6]. All anesthesiologists should therefore have knowledge of DMV, causes of DMV and alternative techniques when mask ventilation has become difficult or has failed [7].

In patients with huge maxillofacial defect, the airway can be secured by blind nasal intubation, oral intubation, fiberoptic intubation or surgical airways. Our patient had a large amount of tissue and muscle deficit on the left 
side of his cheek, perioral edema and chronic osteomyelitis of the mandible. These conditions made proper fitting of the mask difficult and would cause air leakage from the side (Figure 1(a) and Figure 1(b)), and there would also be a risk of spread of infection to the brain. The mouth opening was $<1$ finger, and the use of an oral alternative airway device for intubation was limited. The patient had also received radiation and had distorted airway anatomy, suggesting that DI was predicted. Thus, the airway was pre-evaluated by using 3D-CT of the airway (Figure 1(c)), indicating thatthe orolaryngeal space was narrow but that the epiglottis was placed in the middle. AFOI under conscious sedation was considered to be safe and the only option to avoid complications. According to Benumof et al. [8], AOFI is also the safest approach of the airway management in the patients with predicted DMV. Inadequateventilation occurs in up to $38 \%$ of patients with facial injuries reported by Caplan et al. [9]. Benumof et al. [8] estimated that up to $30 \%$ of deathsare due to inability of successful airway management.

Major challenges during AFOI are providing adequate sedation, maintaining a patent airway and ensuring

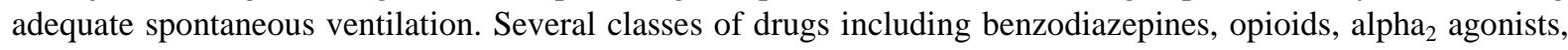
propofol, and ketamine have been reported for conscious sedation during AFOI [2]. Among them, dexmedetomidine is a highly selective, potent alpha $_{2}$ adrenergic receptor agonist. It has the ability to produce profound sedation without causing respiratory depression. In addition, dexmedetomidine decreases salivary secretion through sympatholytic and vagomimetic effects, which is advantageous for fiberoptic intubation [10] [11]. Furthermore, dexmedetomidine provided an optimal intubating condition, less hemodynamic instability and better patient tolerance [12] [13]. Dexmedetomidine thus has many properties that make it suitable for AFOI, and it has been highly recommended for AFOI. In addition to successful use of dexmedetomidine for AFOI in patients with severe submandibular abscess, dexmedetomidine also provided an optimal intubating condition in our patient.

dos Reis Falcao et al. [14] reported a similar type of case with huge facial defect having DMV. They managed the case with transorbital intubation using propofol and fentanyl under spontaneous ventilation. In our case, transorbital intubation was not possible and nasal intubation was required because the plan of surgery was reconstruction of the facial defect. Pre-evaluation of the airway by using 3D-CT provided useful information for planning of airway management.

\section{Conclusion}

In conclusion, for patients who are predicted to have DMV, conscious sedation using dexmedetomidine and AFOI is a good alternative.

\section{References}

[1] El-Orbany, M. and Woehlck, H.J. (2009) Difficult Mask Ventilation. Anesthesia and Analgesia, 109, 1870-1880. http://dx.doi.org/10.1213/ANE.0b013e3181b5881c

[2] Sessler, C.N., Gosnell, M.S., Grap, M.J., Brophy, G.M., O’Neal, P.V., Keane, K.A., Tesoro, E.P. and Elswick, R.K. (2002) The Richmond Agitation-Sedation Scale: Validity and Reliability in Adult Intensive Care Unit Patients. American Journal of Respiratory and Critical Care Medicine, 166, 1338-1344. http://dx.doi.org/10.1164/rccm.2107138

[3] American Society of Anesthesiologists Task Force on Management of the Difficult Airway (2003) Practice Guidelines for Management of the Difficult Airway: An Updated Report by the American Society of Anesthesiologists Task Force on Management of the Difficult Airway. Anesthesiology, 98, 1269-1277. http://dx.doi.org/10.1097/00000542-200305000-00032

[4] Langeron, O., Masso, E., Huraux, C., Guggiari, M., Bianchi, A., Coriat, P. and Riou, B. (2000) Prediction of Difficult Mask Ventilation. Anesthesiology, 92, 1229-1236. http://dx.doi.org/10.1097/00000542-200005000-00009

[5] Asai, T., Koga, K. and Vaughan, R.S. (1998) Respiratory Complications Associated with Tracheal Intubation and Extubation. British Journal of Anaesthesia, 80, 767-775. http://dx.doi.org/10.1093/bja/80.6.767

[6] Johnston, K.D. and Rai, M.R. (2013) Conscious Sedation for Awake Fibreoptic Intubation: A Review of the Literature. Canadian Journal of Anaesthesia, 60, 584-599. http://dx.doi.org/10.1007/s12630-013-9915-9

[7] Kheterpal, S., Han, R., Tremper, K.K., Shanks, A., Tait, A.R., O’Reilly, M. and Ludwig, T.A. (2006) Incidence and Predictors of Difficult and Impossible Mask Ventilation. Anesthesiology, 105, 885-891. http://dx.doi.org/10.1097/00000542-200611000-00007

[8] Benumof, J.L. and Scheller, M.S. (1989) The Importance of Transtracheal Jet Ventilation in the Management of the Difficult Airway. Anesthesiology, 71, 769-778. http://dx.doi.org/10.1097/00000542-198911000-00023 
[9] Caplan, R.A., Posner, K.L., Ward, R.J. and Cheney, F.W. (1990) Adverse Respiratory Events in Anesthesia: A Closed Claims Analysis. Anesthesiology, 72, 828-833. http://dx.doi.org/10.1097/00000542-199005000-00010

[10] Belleville, J.P., Ward, D.S., Bloor, B.C. and Maze, M. (1992) Effects of Intravenous Dexmedetomidine in Humans. I. Sedation, Ventilation, and Metabolic Rate. Anesthesiology, 77, 1125-1133. http://dx.doi.org/10.1097/00000542-199212000-00013

[11] Hall, J.E., Uhrich, T.D., Barney, J.A., Arain, S.R. and Ebert, T.J. (2000) Sedative, Amnestic, and Analgesic Properties of Small-Dose Dexmedetomidine Infusions. Anesthesia and Analgesia, 90, 699-705. http://dx.doi.org/10.1097/00000539-200003000-00035

[12] Chu, K.S., Wang, F.Y., Hsu, H.T., Lu, I.C., Wang, H.M. and Tsai, C.J. (2010) The Effectiveness of Dexmedetomidine Infusion for Sedating Oral Cancer Patients Undergoing Awake Fibreoptic Nasal Intubation. European Journal of Anaesthesiology, 27, 36-40. http://dx.doi.org/10.1097/EJA.0b013e32832e0d2b

[13] Bergese, S.D., Khabiri, B., Roberts, W.D., Howie, M.B., McSweeney, T.D. and Gerhardt, M.A. (2007) Dexmedetomidine for Conscious Sedation in Difficult Awake Fiberoptic Intubation Cases. Journal of Clinical Anesthesia, 19, 141144. http://dx.doi.org/10.1016/j.jclinane.2006.07.005

[14] dos Reis Falcao, L.F., Negreiros, F., Franca, R.F. and Amaral, J.L. (2014) Unusual Access to Airway with Transorbital Intubation. Anesthesiology, 121, 654. http://dx.doi.org/10.1097/ALN.0b013e31829b36af 